\title{
OBTENÇÃO DE CRISTAIS DE LICOPENO A PARTIR DE DESCARTE DE TOMATE ${ }^{1}$
}

\author{
Itaciara L. NUNES ${ }^{2}$, Adriana Z. MERCADANTE ${ }^{2, *}$
}

\section{RESUMO}

As pesquisas com licopeno têm sido intensificadas uma vez que o consumo de alimentos ricos neste caroteno, como tomate e seus produtos, tem sido associado com o menor risco de desenvolvimento de câncer de próstata. Os objetivos deste trabalho foram (1) desenvolver e otimizar um método para extração de licopeno a partir de descarte de tomate, (2) utilizar este método na obtenção de licopeno a partir de diferentes coletas de tomate, (3) obter cristais de licopeno puro e (4) acompanhar o processo de purificação de licopeno por cromatografia líquida de alta eficiência em coluna $\mathrm{C}_{30}$. O método desenvolvido e otimizado através de planejamento experimental fatorial para a obtenção de licopeno consistiu de uma etapa preliminar para retirada de água do tomate, com 4 extrações de 30 minutos, cada uma com 30mL de etanol, seguido por 4 extrações de 120 minutos com acetato de etila e relação massa/volume de 1:0,7. O teor de carotenóides médio (calculado como licopeno) obtido de 6 lotes de tomate foi de 59,2 $\pm 21,8 \mu \mathrm{g} / \mathrm{g}$. Através de duas cristalizações sucessivas obteve-se licopeno com 98\% de pureza. A análise por CLAE mostrou a presença de traços dos isômeros 5-cis, 5-cis, 5'-cis- e 13-cis-licopeno no cristal de licopeno.

Palavras-chave: licopeno; descarte de tomate; extração; cristalização; microscopia; cromatografia líquida de alta eficiência.
\end{abstract}

\section{SUMMARY}

PRODUCTION OF LYCOPENE CRYSTALS FROM TOMATO WASTE. Over the past few years, research regarding lycopene has intensified since the consumption of lycopene rich foods such as tomato and tomato products has been associated with reduced risks of developing prostrate cancer. Thus the objectives of this research were (1) develop and optimise methodology for the extraction of lycopene from tomato waste, (2) use the methodology to obtain lycopene from different tomato batches, (3) produce high purity lycopene crystals and (4) follow the purification process of lycopene using high performance liquid chromatography on a $\mathrm{C}_{30}$ column. The method developed to obtain lycopene from tomato residue, and optimised using a factorial experimental design, consisted of a preliminary water removal step with four 30-minute extractions, each with $30 \mathrm{~mL}$ ethanol, followed by four 120-minute extractions with ethyl acetate, each extraction with a mass/volume ratio of 1:0.7. The mean carotenoid content (calculated as lycopene), obtained from 6 tomato pickings, was $59.2 \pm 21.8 \mu \mathrm{g} / \mathrm{g}$. Lycopene with $98 \%$ purity was obtained from two successive crystallisation processes. HPLC analysis showed the presence of traces of the 5-cis-, 5-cis,5'-cis- and 13-cis-lycopene isomers in the lycopene crystal.

Keywords: lycopene; tomato waste; extraction; microscopy; high performance liquid chromatography.

\section{1 - INTRODUÇÃO}

Os carotenóides são pigmentos naturais, com coloração variando do amarelo ao vermelho, que têm sido largamente utilizados como corantes em alimentos, bebidas, cosméticos e rações animais. Além disso, estes pigmentos possuem comprovada atividade de vitamina A e existem evidências de outras propriedades biológicas, terapêuticas e preventivas de vários tipos de distúrbios e enfermidades em humanos [20,33]. Nos últimos anos, o interesse por licopeno tem aumentado, uma vez que o consumo de alimentos ricos neste caroteno, como tomate e seus produtos, bem como altas concentrações de licopeno no sangue foram associadas ao menor risco de desenvolvimento de câncer de próstata $[12,14,15,24]$.

As principais fontes industriais de carotenóides são a síntese química e a extração a partir de plantas, como urucum (Bixa orellana), e da microalga Dunaliella salina.

\footnotetext{
Recebido para publicação em 06/08/2003. Aceito para publicação em $21 / 06 / 2004$ (001185). (Parte da tese de Doutorado da $1^{a}$ autora, junto ao curso de Pós-graduação em Ciência de Alimentos da FEA/ UNICAMP).

2. Departamento de Ciência de Alimentos - Faculdade de Engenharia de Alimentos, UNICAMP. Cx. Postal 6121, CEP: 13083-970, Campinas-SP. E-mail: azm@fea.unicamp.br.

* A quem a correspondência deve ser enviada.
}

Várias empresas comercializam alguns padrões de carotenóides para fins analíticos. Entretanto, estes padrões possuem um alto custo e vida-útil reduzida, uma vez que após abertura da embalagem ocorre formação de isômeros e produtos de oxidação devido ao longo sistema de ligações duplas conjugadas presente nos carotenóides [6].

Os trabalhos encontrados na literatura, em sua maioria, enfocam a extração e análise quantitativa de carotenóides, geralmente realizada em equipamentos de pequeno porte, como liqüidificador, homogeneizador e agitador para ruptura mecânica, utilizando pequena quantidade de amostra e acetona como solvente para extração de licopeno [17, 18, 28, 29].

CEREZAL \& PIÑERA [8] relataram diferentes métodos de obtenção de carotenóides a partir de resíduos de frutas cítricas, utilizando solventes orgânicos (acetona ou hexano) ou d-limoneno, seguida ou não de saponificação, neutralização com fosfato ácido de potássio e precipitação com iso-propanol ou cromatografia de coluna aberta de permeação em gel. Dependendo do resíduo utilizado, o rendimento variou de 23 a 100\%.

Extração seletiva em duas etapas, a $50^{\circ} \mathrm{C}$ em "shaker", de carotenóides e compostos de pungência (capsaicinóides) a partir de pimenta seca moída foi avaliada por SANTAMARÍA et al. [26]. Na primeira etapa, com $30 \%$ de etanol aquoso, foram extraídos $62 \%$ de 
capsaicinóides e houve pequena remoção de carotenóides $(1,4 \%)$. Na extração subseqüente com etanol comercial foram recuperados $26 \%$ de capsaicinóides e $70 \%$ de carotenóides. Quando foi realizado um pré-tratamento com enzimas celulolíticas e pectinolíticas houve um aumento para 83\% na recuperação de carotenóides.

Extração de licopeno com óleo de girassol a partir de resíduos do processamento de pasta e de suco de tomate previamente submetidos a tratamento térmico $\left(100-125^{\circ} \mathrm{C}\right.$ ) e saponificação com hidróxido de potássio etanólico, foi descrita em uma patente russa [13].

Diante dos fatos expostos acima e considerando que o descarte de vegetais e frutas na CEASA - Campinas (Centrais de Abastecimento de Campinas S/A) atinge em média 7,8 mil toneladas/ano, este trabalho teve como objetivos (1) desenvolver e otimizar um método simples para extração de licopeno a partir de descarte de tomate, (2) utilizar este método na obtenção de licopeno a partir de diferentes coletas de tomate, (3) obter cristais de licopeno com alto grau de pureza através de procedimento de baixo custo e (4) acompanhar o processo de purificação de licopeno por cromatografia líquida de alta eficiência (CLAE) em coluna $\mathrm{C}_{30}$.

\section{2 - MATERIAL E MÉTODOS}

\section{1 - Amostras}

As amostras de descarte de tomate $(\cong 5 \mathrm{Kg})$ foram coletadas na CEASA-Campinas, Brasil, após uma préseparação manual de jornais, caixas, outros materiais recicláveis e orgânicos, durante o período de junho de 2002 a março de 2003, totalizando 6 lotes.

\section{2 - Medidas físico-químicas}

Após a esterilização dos tomates $\left(121^{\circ} \mathrm{C} / 15 \mathrm{~min}\right)$ a amostra foi homogeneizada, colocada em cubeta $(2 \mathrm{~cm})$ e realizada avaliação de cor em colorímetro Hunter (modelo ColorQuest II) com calibração RSIN (Reflectância Especular Incluída), iluminante D65 e ângulo de observação de $10^{\circ}$, utilizando-se a escala CIELAB. Foram realizadas seis leituras de cor de cada amostra e calculada a média e o desvio padrão dos parâmetros luminosidade ( $\left.\mathrm{L}^{*}\right)$, índice de saturação vermelho $\left(\mathrm{a}^{*}\right)$ e índice de saturação amarelo (b*). Os valores de Chroma e hue foram calculados segundo ARIAS et al. [2].

As medidas de $\mathrm{pH}$ foram realizadas em pHmêtro Tecnal (modelo Tec-2); os valores de ${ }^{\circ}$ Brix foram obtidos em refratômetro Atago (modelo 8572) e corrigidos utilizando-se a tabela de correção de temperatura para $20^{\circ} \mathrm{C}$. Todas as análises foram realizadas em triplicata, de acordo com a AOAC [1].

\section{3 - Obtenção do extrato rico em licopeno}

\subsection{1 - Retirada de água}

Testes preliminares mostraram a necessidade de um pré-tratamento para retirada da água presente no tomate antes da extração dos carotenóides. Através de um planejamento fatorial completo $2^{4}$, com pontos centrais, testou-se tipo de solvente (metanol e etanol), volume de solvente (30 e 60mL), bem como tempo (30 e 60min) e número de extrações ( 2 e 4) para que o extrato final ficasse livre de água. Esta etapa foi realizada em mesa agitadora (Tecnal, TE-140) à temperatura ambiente, utilizando $30 \mathrm{~g}$ de amostra. O álcool contendo água foi descartado.

A retirada de água foi seguida de extração com acetato de etila e concentração em evaporador rotatório $\left(\mathrm{T}<35^{\circ} \mathrm{C}\right)$, verificando-se a presença ou não de água residual no extrato. A resposta, percentual de água no extrato, foi determinada por diferença de peso, antes e após secagem do extrato $\left(110^{\circ} \mathrm{C}\right.$ em estufa até peso constante).

Todos os experimentos foram conduzidos em duplicata.

\subsection{2 - Extração de licopeno}

Após testes preliminares, verificou-se que os solventes com maior potencial de extração de licopeno foram diclorometano, acetona e acetato de etila. Porém, para verificar as variáveis significativas na extração de licopeno foram utilizados como solventes o acetato de etila e etanol comercial, tendo em vista que o diclorometano é um solvente clorado, e, assim como a acetona, controlado pela Polícia Federal no Brasil. Na época do desenvolvimento deste trabalho, o acetato de etila não era controlado. A escolha do etanol se deve ao seu baixo custo no Brasil.

Foram realizados dois planejamentos fatoriais fracionários $2^{4-1}$, um utilizando acetato de etila como solvente de extração e outro etanol, para verificar os efeitos de alguns fatores na concentração do extrato rico em licopeno (Tabela 1). Todos os experimentos foram conduzidos em duplicata em mesa agitadora (150rpm) à temperatura ambiente.

TABELA 1. Fatores e níveis estudados na etapa de extração de licopeno.

\begin{tabular}{lcc}
\hline \multirow{2}{*}{ Fatores } & \multicolumn{2}{c}{ Níveis } \\
\cline { 2 - 3 } & -1 & +1 \\
\hline Número de extraçães & 2 & 4 \\
Esterilização & com & sem \\
Relação massa de tomate/volume de solvente & $1: 0,7$ & $1: 2$ \\
Tempo de extração & $30 \mathrm{~min}^{\star} / 20 \mathrm{~h}^{\star \star}$ & $120 \mathrm{~min}^{\star} / 24 \mathrm{~h}^{\star \star}$ \\
\hline
\end{tabular}

*tempo de extração com acetato de etila
**tempo de extração com etanol

Para otimização das condições de extração foi, posteriormente, realizado um planejamento $2^{2}$ completo com pontos centrais e axiais somente com os fatores significativos.

A quantificação dos extratos foi realizada em espectrofotômetro Beckman (modelo DU-70) através da leitura no comprimento de onda de máximo de absor- 
ção em éter de petróleo e a concentração de licopeno foi calculada utilizando absortividade de 3450 [9].

\section{4 - Purificação do extrato rico em licopeno}

Devido ao alto custo das fases estacionárias para cromatografia, escolheu-se a cristalização como método para purificação de licopeno.

O extrato foi dissolvido em diclorometano/etanol (1:4) a temperatura de $50-60^{\circ} \mathrm{C}$, colocado em banho de gelo para abaixamento gradual da temperatura e em seguida levado ao freezer por uma noite para a formação dos cristais. Os cristais foram filtrados em papel Whatman Chr 3mm, lavados com etanol gelado, e secos em estufa a vácuo à temperatura ambiente. O procedimento de cristalização foi novamente realizado para obtenção de cristais com maior teor de pureza.

\section{5 - Análise de carotenóides por CLAE}

Foi utilizado um cromatógrafo líquido de alta eficiência com sistema quaternário de bombeamento de solventes (Waters, modelo controller 600), detector de arranjo de diodos (Waters, modelo 996), injetor "Rheodyne" com alça de amostra de $20 \mu \mathrm{L}$, forno externo com controle de temperatura, desgaseificador "on line" e sistema de aquisição e processamento de dados Millenium (Waters, versão 3.05.01). Os espectros foram adquiridos entre 250 e $600 \mathrm{~nm}$ e os cromatogramas processados no comprimento de onda máximo $\left(\lambda_{\max }\right)$ de cada carotenóide.

Os carotenóides foram separados em coluna $\mathrm{C}_{30}$ polimérica da marca YMC ( $3 \mu \mathrm{m}, 250$ x 4,6mm) com MeOH $(0,1 \%$ trietilamina (TEA))/éter $t$-metil butílico (TBME) (50:50) em modo isocrático a $1 \mathrm{~mL} / \mathrm{min}$ como fase móvel e temperatura da coluna mantida a $33^{\circ} \mathrm{C}$.

Todos os solventes utilizados foram grau cromatográfico e previamente filtrados em sistema Millipore de filtração a vácuo, com membrana para solvente orgânico de $0,45 \mu \mathrm{m}$. Todas as amostras foram filtradas em membrana de polietileno com $0,22 \mu \mathrm{m}$ de poro.

A caracterização dos carotenóides foi baseada nos seguintes parâmetros: (1) ordem de eluição na coluna $\mathrm{C}_{30}$, (2) comparação das características dos espectros no UV-visivel ( $\lambda_{\max }$, grau de estrutura fina (\%III/II) e intensidade do pico cis $\left(\% \mathrm{~A}_{\mathrm{B}} / \mathrm{A}_{\mathrm{II}}\right)$ ) com dados da literatura $[7,19,23]$ e (3) comparação com padrões de alltrans- $\gamma$-caroteno, all-trans- $\beta$-caroteno, 5-cis-, 9-cis-, 13cis- e all-trans-licopeno cedidos pela Roche Vitamins (Suíça).

\section{6 - Análises microscópicas}

\subsection{1 - Microscopia óptica}

Amostras de cristais de licopeno natural e licopeno sintético (Roche Vitamins) foram montadas individualmente sobre lâminas, em óleo de silicone, óleo de imersão ou a seco e cobertas com lamínula e observadas ao microscópio óptico (Carlzeiss, modelo Jenaval), sob luz normal ou polarizada.
As imagens geradas no microscópio óptico foram capturadas por câmera utilizando-se o Software Global Lab Image 3.0.

\subsection{2 - Microscopia eletrônica de varredura}

As amostras mencionadas acima foram aspergidas sobre fita metálica adesiva de dupla face, colocadas sobre suportes metálicos e recobertas com ouro (240s/40mA) em metalizador a vácuo (Balzers). As micrografias foram obtidas no microscópio Jeol, modelo T-300, sob aceleração de $15 \mathrm{KV}$ através de captura de imagem.

\section{7 - Análise estatística}

Os planejamentos experimentais foram analisados pelo programa Statistica 5.0 [31].

\section{3 - RESULTADOS E DISCUSSÃO}

\section{1 - Condições otimizadas para obtenção do ex- trato rico em licopeno}

Os resultados do planejamento experimental fatorial, apresentados na Tabela 2, mostraram uma eficiência de 1 a $75 \%$ na retirada de água do tomate.

Dos fatores avaliados, o volume de solvente e tempo de extração não tiveram efeito significativo na resposta (Tabela 3), e conseqüentemente foram fixados nos seus níveis inferiores. Por outro lado, o efeito do tipo de solvente e número de extrações foi significativo, sendo o etanol e 4 extrações as melhores condições para retirada de água, como pode ser verificado nos ensaios 6, 8 e 14 (Tabela 2).

TABELA 2. Condições experimentais do planejamento fatorial completo e respostas para a etapa de retirada de água.

\begin{tabular}{|c|c|c|c|c|c|c|}
\hline \multirow[b]{2}{*}{ Ensaios } & \multicolumn{4}{|c|}{ Fatores } & \multicolumn{2}{|c|}{ Respostas } \\
\hline & Solvente & $\begin{array}{l}\text { Volume de } \\
\text { solvente }(\mathrm{mL})\end{array}$ & $\begin{array}{l}\text { Número de } \\
\text { extrações }\end{array}$ & $\begin{array}{c}\text { Tempo de } \\
\text { extração (min) }\end{array}$ & R1 & $\mathrm{R} 2$ \\
\hline 1 & $\mathrm{MeOH}$ & 30 & 2 & 30 & 94,5 & 94,4 \\
\hline 2 & EtOH & 30 & 2 & 30 & 96,3 & 95,0 \\
\hline 3 & $\mathrm{MeOH}$ & 60 & 2 & 30 & 94,2 & 94,9 \\
\hline 4 & EtOH & 60 & 2 & 30 & 91,4 & 88,3 \\
\hline 5 & $\mathrm{MeOH}$ & 30 & 4 & 30 & 84,0 & 81,7 \\
\hline 6 & $\mathrm{EtOH}$ & 30 & 4 & 30 & 25,5 & 26,3 \\
\hline 7 & $\mathrm{MeOH}$ & 60 & 4 & 30 & 39,6 & 43,5 \\
\hline 8 & $\mathrm{EtOH}$ & 60 & 4 & 30 & 35,7 & 32,8 \\
\hline 9 & $\mathrm{MeOH}$ & 30 & 2 & 60 & 97,4 & 95,9 \\
\hline 10 & $\mathrm{EtOH}$ & 30 & 2 & 60 & 98,5 & 99,0 \\
\hline 11 & $\mathrm{MeOH}$ & 60 & 2 & 60 & 96,1 & 97,2 \\
\hline 12 & EtOH & 60 & 2 & 60 & 94,6 & 97,7 \\
\hline 13 & $\mathrm{MeOH}$ & 30 & 4 & 60 & 41,6 & 43,3 \\
\hline 14 & EtOH & 30 & 4 & 60 & 27,0 & 24,8 \\
\hline 15 & $\mathrm{MeOH}$ & 60 & 4 & 60 & 29,1 & 28,6 \\
\hline 16 & $\mathrm{EtOH}$ & 60 & 4 & 60 & 44,5 & 44,0 \\
\hline 17 & $\mathrm{MeOH}$ & 45 & 3 & 45 & 85,4 & 84,9 \\
\hline 18 & $\mathrm{MeOH}$ & 45 & 3 & 4 & 81,5 & 82,8 \\
\hline 19 & $\mathrm{MeOH}$ & 45 & 3 & 45 & 84,3 & 83,3 \\
\hline 20 & EtOH & 45 & 3 & 45 & 56,1 & 55,9 \\
\hline 21 & $\mathrm{EtOH}$ & 45 & 3 & 45 & 54,3 & 55,3 \\
\hline 22 & EtOH & 45 & 3 & 45 & 57,5 & 56,8 \\
\hline
\end{tabular}


TABELA 3. Efeitos dos fatores na etapa de retirada de água do tomate.

\begin{tabular}{lcc}
\hline & Efeitos & $p$ \\
\hline Solvente & $-13,65^{*}$ & 0,00 \\
Volume de solvente $(\mathrm{mL})$ & $-4,56$ & 0,15 \\
Número de extrações & $-54,60^{*}$ & 0,00 \\
Tempo de extração $(\min )$ & $-3,66$ & 0,25 \\
\hline
\end{tabular}

*p<0,05 (estatisticamente significativo a 95\% de confiança)

Portanto, nos níveis estudados, as melhores condições de retirada de água do tomate foram obtidas quando foram realizadas 4 extrações de 30 minutos cada, utilizando $30 \mathrm{~mL}$ de etanol por extração.

HAKALA \& HEINONEN [17] adicionaram $\mathrm{Na}_{2} \mathrm{SO}_{4}$ como dessecante junto com os solventes testados, éter de petróleo e acetona, para extrair licopeno de purê de tomate. Entretanto, os autores não mencionaram a quantidade de dessecante acrescentada.

Quanto à extração de licopeno, através dos resultados obtidos a partir dos planejamentos experimentais fracionários $2^{4-1}$ (Tabela 4), foi possivel verificar que

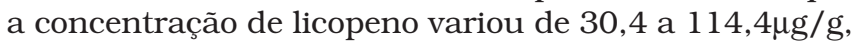
quando acetato de etila foi utilizado como solvente e de 2,6 a $14,6 \mu \mathrm{g} / \mathrm{g}$ com etanol, mostrando que a extração com acetato de etila foi significativamente mais eficiente.

TABELA 4. Condições experimentais dos planejamentos fatoriais fracionários e respostas para a etapa de extração de carotenóides com etanol e acetato de etila.

\begin{tabular}{|c|c|c|c|c|c|c|c|c|}
\hline \multirow[b]{2}{*}{ Ensaios } & \multicolumn{4}{|c|}{ Fatores } & \multicolumn{2}{|c|}{ Respostas $^{a}$} & \multicolumn{2}{|c|}{ Respostas $^{0}$} \\
\hline & $\begin{array}{c}\mathrm{N}^{0} \text {. de } \\
\text { extraçöes }\end{array}$ & Esterilização & $\begin{array}{c}\text { Relação } \\
\text { massa/volume }\end{array}$ & $\begin{array}{c}\text { Tempo de } \\
\text { extração } \\
\min ^{a} \text { ou horas }{ }^{b}\end{array}$ & R1 & R2 & R1 & R2 \\
\hline 1 & 2 & Sim & $1: 0,7$ & $30^{\circ}$ ou $20^{\circ}$ & 30,4 & 31,3 & 2,6 & 2,7 \\
\hline 2 & 4 & Sim & $1: 0,7$ & $120^{a}$ ou $24^{b}$ & 114,4 & 102,5 & 8,2 & 7,4 \\
\hline 3 & 2 & Não & $1: 0,7$ & $120^{\mathrm{a}}$ ou $24^{\mathrm{b}}$ & 46,5 & 47,2 & 5,00 & 4,8 \\
\hline 4 & 4 & Não & $1: 0,7$ & $30^{a}$ ou $20^{b}$ & 53,7 & 50,1 & 6,0 & 5,7 \\
\hline 5 & 2 & Sim & $1: 2$ & $120^{\mathrm{a}}$ ou $24^{\mathrm{b}}$ & 85,2 & 83,8 & 8,0 & 7,9 \\
\hline 6 & 4 & Sim & $1: 2$ & $30^{a}$ ou $20^{b}$ & 52,9 & 55,1 & 10,8 & 10,3 \\
\hline 7 & 2 & Não & $1: 2$ & $30^{\mathrm{a}}$ ou $20^{\mathrm{b}}$ & 44,1 & 45,7 & 7,1 & 6,8 \\
\hline 8 & 4 & Não & $1: 2$ & $120^{\mathrm{a}}$ ou $24^{\mathrm{b}}$ & 58,9 & 56,8 & 14,3 & 14,6 \\
\hline
\end{tabular}

Quando etanol foi utilizado para a extração, todos os fatores no nivel superior acarretaram aumento significativo na concentração de licopeno (Tabela 5), como mostram os ensaios 2,6 e 8 da Tabela 4 .

Para a extração com acetato de etila, o tempo e o número de extrações foram também significativos ( $T a-$ bela 5) levando a um aumento na concentração de licopeno quando em seu nível superior (ensaios 2 e 8 da Tabela 4). A relação massa/volume não apresentou efeito significativo.

O uso de esterilização também teve um efeito significativo implicando em maior extração de licopeno, tanto com acetato de etila como com etanol (Tabela 5). Este fato já era esperado uma vez que o processamento térmico pode ocasionar a ruptura da parede celular e conseqüente redução das forças de interação entre o licopeno e a matriz tecidual, tornando o pigmento mais acessivel à extração [30].

TABELA 5. Efeitos dos fatores para a etapa de avaliação das condições de extração de carotenóides utilizando acetato de etila ou etanol.

\begin{tabular}{lccccc}
\hline & \multicolumn{2}{c}{ Etanol } & & \multicolumn{2}{c}{ Acetato de etila } \\
\cline { 2 - 3 } \cline { 6 - 6 } & Efeitos & $\mathrm{p}$ & & Efeitos & $\mathrm{p}$ \\
\hline $\mathrm{N}^{0}$ extrações & $4,05^{*}$ & 0,00 & & 16,28 & 0,07 \\
Esterilização & 0,81 & 0,06 & & $-19,07^{*}$ & 0,04 \\
Relação massa/volume & $4,68^{*}$ & 0,00 & & 0,79 & 0,92 \\
Tempo (min) & $2,28^{*}$ & 0,00 & & $28,99^{*}$ & 0,00 \\
\hline
\end{tabular}

* $\mathrm{p}<0,05$ (estatisticamente significativo a 95\% de confiança)

A extração foi otimizada através de um novo planejamento fatorial completo $2^{2}$ aumentando o número de extrações e o tempo de cada extração (Tabela 6), sendo fixados os fatores não significativos em seus níveis inferiores (relação massa/volume de 1:0,7 e uso de esterilização) e acetato de etila como solvente de extração.

TABELA 6. Condições experimentais do planejamento fatorial completo e respostas para a etapa de otimização da extração de carotenóides com acetato de etila.

\begin{tabular}{|c|c|c|c|c|}
\hline \multirow[b]{2}{*}{ Ensaios } & \multicolumn{2}{|c|}{ Fatores } & \multicolumn{2}{|c|}{ Respostas } \\
\hline & $\begin{array}{c}\text { Tempo de } \\
\text { extração (min) }\end{array}$ & $\begin{array}{l}\text { Número de } \\
\text { extrações }\end{array}$ & $\mathrm{R} 1$ & $\mathrm{R} 2$ \\
\hline 1 & 60 & 2 & 48,55 & 52,03 \\
\hline 2 & 180 & 2 & 84,93 & 81,59 \\
\hline 3 & 60 & 6 & 83,77 & 83,04 \\
\hline 4 & 180 & 6 & 100,43 & 92,90 \\
\hline 5 & 50 & 4 & 47,75 & 49,56 \\
\hline 6 & 205 & 4 & 80,87 & 87,39 \\
\hline 7 & 120 & 1 & 27,93 & 26,99 \\
\hline 8 & 120 & 7 & 110,4 & 108,12 \\
\hline 9 & 120 & 4 & 100,43 & 95,51 \\
\hline 10 & 120 & 4 & 104,06 & 104,93 \\
\hline
\end{tabular}

Resposta: concentração ( $\mu \mathrm{g} / \mathrm{g})$ de carotenóides totais, calculada como licopeno.

Na curva de nível, apresentada na Figura 1, podese verificar três pontos na região ótima de extração de licopeno. Estes pontos ótimos apresentam diferentes condições: 4 extrações de 120 minutos, 7 extrações de 120 minutos e 6 extrações de 180 minutos, obtendo-se cerca de $100 \mu \mathrm{g}$ de licopeno/g de tomate esterilizado. 
Conseqüentemente, foi escolhido, como a melhor condição, o uso de 4 extrações de 120 minutos, tendo em vista o menor tempo total de análise e o menor gasto de solvente.

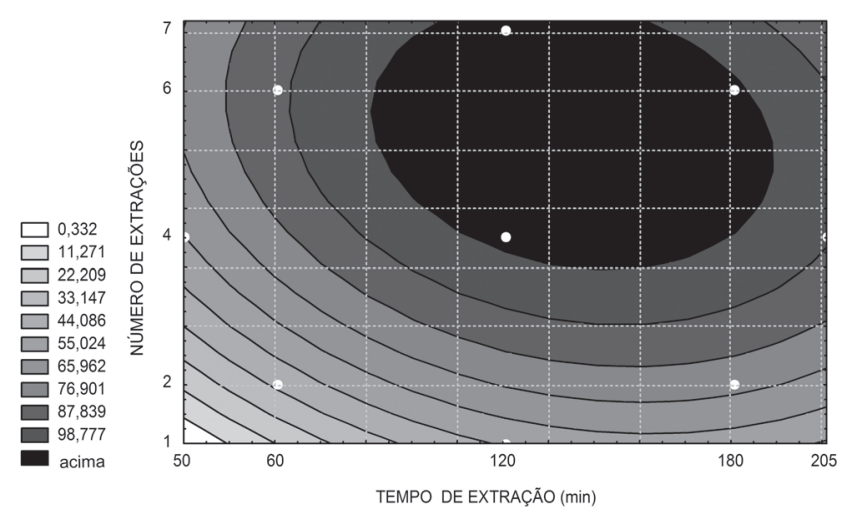

FIGURA 1. Curva de nível para a concentração de carotenóides totais $(\mu \mathrm{g} / \mathrm{g})$, em função do número de extrações e tempo de extração.

Não foi possível comparar a eficiência do método desenvolvido no presente estudo com a literatura, pois nenhum dos métodos disponíveis para obtenção de licopeno foi desenvolvido através de planejamentos fatoriais. Cabe esclarecer que o objetivo deste trabalho foi obter maior quantidade de licopeno com o menor gasto de solvente. Deste modo, em nenhum momento foi feita extração exaustiva de carotenóides, e a maior parte das xantofilas foi descartada com o etanol na etapa de retirada de água.

\section{2 - Cristais de licopeno}

As Figuras 2 e 3 apresentam, respectivamente, os cromatogramas do extrato rico em licopeno e dos cristais obtidos após a $1^{\mathrm{a}}$ e $2^{\mathrm{a}}$ cristalização. A Tabela 7 mostra a identidade dos picos presentes nestas figuras.

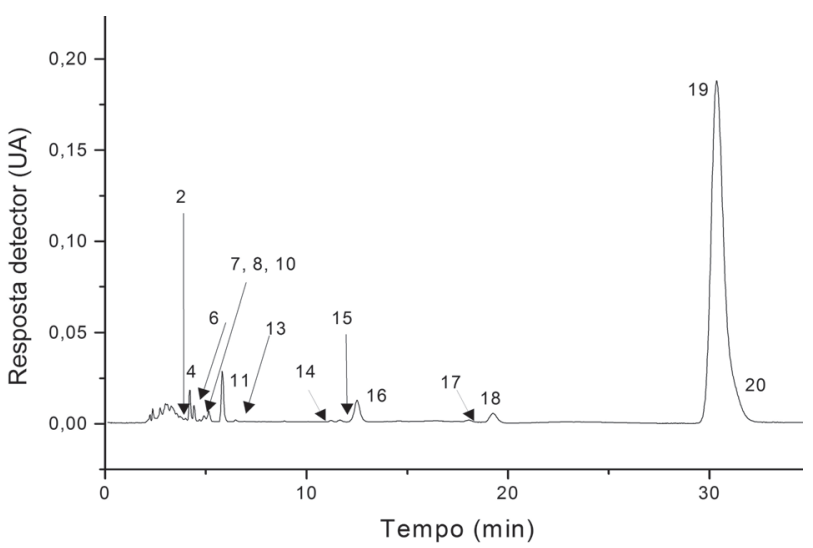

FIGURA 2. Cromatograma obtido por CLAE do extrato rico em licopeno. Condições: coluna $\mathrm{C}_{30} \mathrm{YMC}$, fase móvel $\mathrm{MeOH}(0,1 \%$ TEA)/TBME (50:50), fluxo de $1 \mathrm{~mL} / \mathrm{min}$ e temperatura da coluna de $33^{\circ} \mathrm{C}$. Processamento no $\lambda_{\max }$. A identidade dos picos está apresentada na Tabela 7.
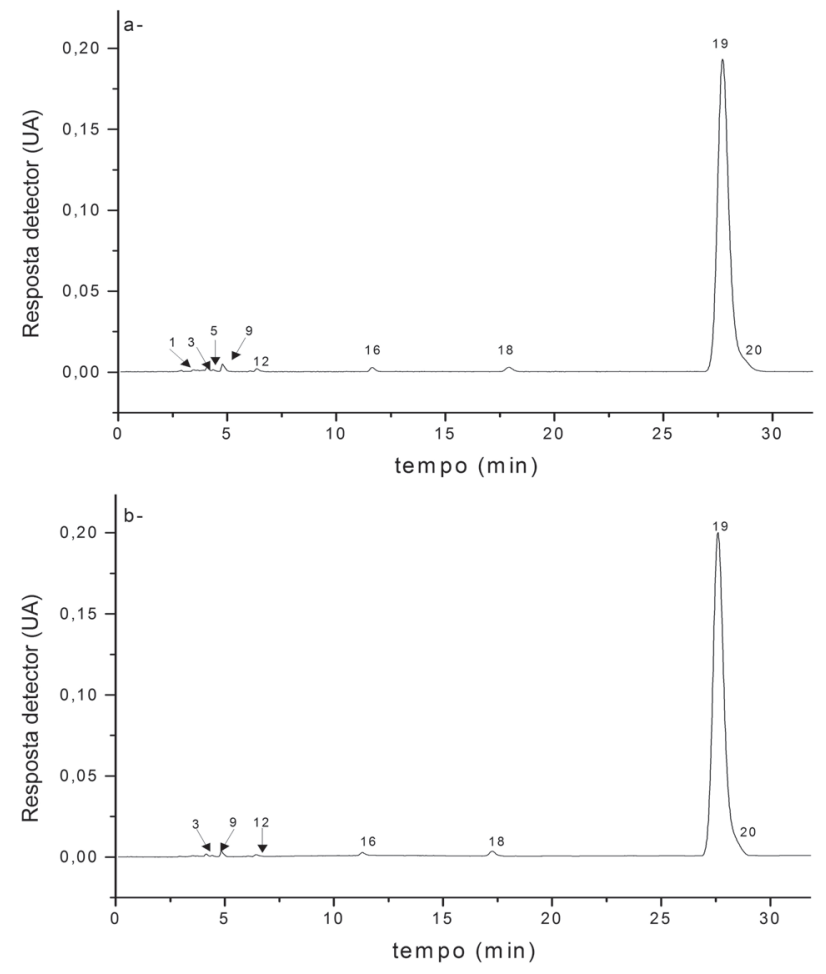

FIGURA 3. Cromatograma, obtido por CLAE, dos cristais de licopeno após: a- $1^{\underline{a}}$ cristalização, b- $2^{\underline{a}}$ cristalização. Condições: coluna $\mathrm{C}_{30}$, fase móvel $\mathrm{MeOH}(0,1 \% \mathrm{TEA}) / \mathrm{TBME}$ (50:50), fluxo de $1 \mathrm{~mL} / \mathrm{min}$ e temperatura da coluna de $33^{\circ} \mathrm{C}$. Processamento no $\lambda_{\max }$. A identidade dos picos está apresentada na Tabela 7.

TABELA 7. Principais características, obtidas por CLAE, dos carotenóides presentes no extrato rico em licopeno e nos cristais de licopeno.

\begin{tabular}{clcccc}
\hline $\mathrm{N}^{\circ}$ pico $^{\mathrm{a}}$ & Carotenóides & $\mathrm{t}_{\mathrm{R}}(\mathrm{min})$ & $\lambda_{\max }(\mathrm{nm})^{\mathrm{b}}$ & $\%$ III/II & $\% \mathrm{~A}_{\mathrm{B}} / \mathrm{A}_{\|}$ \\
\hline 1 & mistura & 3,5 & $385,405,429$ & - & - \\
2 & mistura & 4,0 & $431,454,485$ & - & - \\
3 & não identificado & 4,1 & $404,424,448$ & 20 & 0 \\
4 & mistura & 4,2 & $403,428,452$ & - & - \\
5 & não identificado & 4,3 & $411,433,460$ & 57 & 0 \\
6 & cis-fitoflueno & 4,4 & $332,347,365$ & 58 & - \\
7 & fitoflueno & 4,7 & $332,347,365$ & 71 & - \\
8 & não identificado & 4,9 & $277,344,434,456$ & 25 & 20 \\
9 & não identificado & 5,0 & $343,412,435,463$ & 61 & 15 \\
10 & não identificado & 5,1 & $339,(423), 449,(471)$ & 0 & 39 \\
11 & all-trans- $\beta$-caroteno & 5,8 & $(423), 452,479$ & 27 & 0 \\
12 & all-trans- $\delta$-caroteno & 6,4 & $345,433,457,487$ & 70 & 0 \\
13 & mistura & 6,5 & $337,(416), 447,474$ & - & - \\
14 & 15-cis-licopeno & 11,2 & $359,443,468,500$ & 50 & 75 \\
15 & all-trans- $\gamma$-caroteno & 11,5 & $349,435,460,489$ & 50 & 0 \\
16 & 13-cis-licopeno & 12,5 & $359,440,465,496$ & 48 & 52 \\
17 & 9-cis-licopeno & 18,1 & $361,440,466,498$ & 69 & 13 \\
18 & 5-cis,5'-cis-licopeno & 19,3 & $361,445,471,503$ & 65 & 8 \\
19 & all-trans-licopeno & 30,4 & $362,446,472,503$ & 74 & 6 \\
20 & 5-cis-licopeno & 31,2 & $362,446,472,503$ & 74 & 6 \\
\hline
\end{tabular}

a Numeração de acordo com os cromatogramas das Figuras 2 e 3 .
bS Solvente: metanol (0,1\%trietilamina)/éter tert-metil butílico (50:50) 
Os isômeros 15-cis- (pico 14), 13-cis- (pico 16), 9cis- (pico 17) e 5-cis-licopeno (pico 20) foram identificados por comparação da ordem de eluição em coluna $\mathrm{C}_{30}$ com a literatura [10, 21, 25], e dos $\lambda_{\max }$ e forma do espectro com dados previamente relatados [19, 23, 27]. A identidade destes isômeros foi confirmada com padrões.

Comparando as informações do espectro de absorção com a literatura [27] e considerando que a intensidade do pico cis é menor para os isômeros em posição próxima dos grupos terminais, o pico 18 foi tentativamente identificado como 5-cis,5'-cis-licopeno. À medida que a intensidade do pico cis vai aumentando (Tabela 7), a isomeria está provavelmente localizada mais ao centro da cadeia poliênica, como nos isômeros 9-cis- (pico 17), 13-cis- (pico 16) e 15-cis-licopeno (pico 14).

$\mathrm{O}$ all-trans- $\beta$-caroteno (pico 11) e all-trans- $\gamma$-caroteno (pico 15) apresentaram características espectrais similares às relatadas na literatura [7, 23] e às dos padrões autênticos, inclusive o mesmo tempo de retenção. As propriedades dos espectros do all-trans- $\delta$-caroteno (pico 12) e do all-trans-fitoflueno (pico 7) foram similares às encontradas por BRITTON [7]. Como esperado para carotenóides com 5 ligações duplas conjugadas, o cisfitoflueno (pico 6) apresentou $\lambda_{\max }$ igual ao isômero trans e menor grau de estrutura fina.

Utilizando coluna $\mathrm{C}_{30}$, de 4 a 10 isômeros cis de licopeno foram separados em vários trabalhos [4, 5, 11], entretanto, com exceção do all-trans-licopeno $[4,5,11]$ e do prolicopeno [11], os demais isômeros não foram identificados nestes estudos.

No extrato rico em licopeno obtido no presente estudo, o all-trans-licopeno perfez $71 \%$ da área dos carotenóides. Após a $1^{\text {a }}$ cristalização, a porcentagem de pureza ficou em torno de $96 \%$ e com a $2^{\text {a }}$ cristalização atingiu 98\% de pureza, semelhante à pureza do padrão de licopeno sintético (Roche Vitamins) que foi de 99\%.
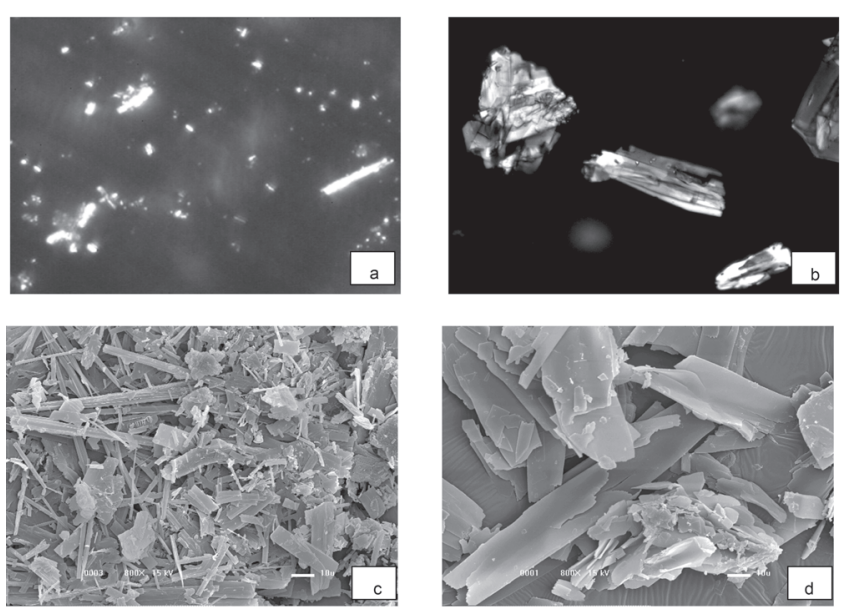

FIGURA 4. Microscopia óptica: a) cristais de licopeno natural sob luz polarizada, b) cristais de licopeno sintético sob luz polarizada. Microscopia eletrônica de varredura: c) cristais de licopeno natural, d) cristais de licopeno sintético. Aumento 800x.
HAKALA \& HEINONEN [17], utilizando Sep-pak de sílica e CLAE com coluna $\mathrm{C}_{18}$ como método de purificação de licopeno, atingiram $97 \%$ de pureza somente após a terceira cromatografia semi-preparativa.

Como alternativa à extração com solventes e purificação, GÓMEZ-PRIETO et al. [16] utilizaram $\mathrm{CO}_{2}$ com fluxo de $4 \mathrm{~mL} / \mathrm{min}$ a $40^{\circ} \mathrm{C}$ sem modificador, obtendo um extrato com $88 \%$ de all-trans-licopeno e $12 \%$ de cislicopeno a partir de tomate liofilizado.

Tanto os cristais de licopeno natural obtidos neste trabalho, como os sintéticos, apresentaram formas cristalinas irregulares, predominando as formas prismáticas alongadas. Os cristais sintéticos atingiram cerca de $3,25 \mu \mathrm{m}$ (Figuras $4 b e 4 d$ ) e os naturais chegaram a medir $1,75 \mu \mathrm{m}$ (Figura $4 c$ ), mas nestes predominaram cristais com tamanho abaixo de $1 \mu \mathrm{m}$ (Figura $4 a$ ).

\section{3 - Teores de licopeno em descarte de tomate}

Nas amostras de descarte de tomate cru os valores médios de $\mathrm{pH}$ variaram de 4,41 a 4,61 e entre 4,32 e 4,50 para as amostras esterilizadas. Os valores de ${ }^{\circ} \mathrm{Brix}$ ficaram entre 3,8 e 4,0 para ambas as amostras. Estes valores se encontram dentro da faixa de $\mathrm{pH}(4,4$ a 4,6) e ${ }^{\circ}$ Brix $(3,8$ a 4,6) reportada para tomate cru [32].

Através dos valores apresentados na Tabela 8 podese verificar uma variação de 28,4 a $93,5 \mu \mathrm{g} / \mathrm{g}$ na concentração de carotenóides totais (calculado como licopeno) entre os 6 lotes de amostras coletados no período de 10 meses. O teor muito menor de licopeno da amostra coletada em março pode estar relacionado à alta precipitação pluviométrica ocorrida nos 3 meses anteriores (CEPAGRI, comunicação pessoal). Embora seja comprovado que a concentração de carotenóides varia em função da variedade/cultivar, época do ano e localização geográfica [3, 18, 22], não é possível fazer esta relação no presente estudo uma vez que foi utilizado descarte de tomate onde não há qualquer controle destas variáveis.

O teor médio de carotenóides, obtido no presente estudo, 59,2 $\pm 21,8 \mu \mathrm{g} / \mathrm{g}$, foi maior do que o encontrado em tomate $(41,4 \pm 20,1 \mu \mathrm{g} / \mathrm{g})$ cultivar Santa Cruz comercializado em Campinas, Brasil [32]. YANG et al. [34] encontraram teor de $26,1 \mu \mathrm{g} / \mathrm{g}$ em tomate vermelho. A concentração de licopeno em tomate analisado na Finlândia variou de 38 a $66 \mu \mathrm{g} / \mathrm{g}$ no período de verão, enquanto no período de outubro a março (outono) os teores encontrados foram de 26 a $31 \mu \mathrm{g} / \mathrm{g}$ [18]. Cabe lembrar que nestes três trabalhos a extração foi quantitativa, enquanto que em nosso estudo a extração não foi exaustiva.

Os valores de parâmetros de cor dos tomates esterilizados estão apresentados na Tabela 8 . No presente estudo, o parâmetro a* foi o que apresentou as mais altas correlações com a concentração de licopeno $(0,84)$, quando utilizada regressão linear e 0,86 , quando utilizada regressão exponencial. A correlação entre teor de licopeno e os valores dos demais parâmetros foi menor que 0,80 , tanto para regressão linear como exponencial. 
TABELA 8. Teores de licopeno $(\mu \mathrm{g} / \mathrm{g})$ e parâmetros Cielab de cor dos tomates esterilizados coletados em diferentes épocas do ano.

\begin{tabular}{|c|c|c|c|c|c|c|c|c|}
\hline $\begin{array}{l}\text { Período de coleta das } \\
\text { amostras }\end{array}$ & $\begin{array}{c}\text { licopeno } \\
(\mu \mathrm{g} / \mathrm{g})^{\mathrm{a}}\end{array}$ & $L^{*}$ & $a^{*}$ & $b^{*}$ & $a^{*} / b^{*}$ & $\left(a^{\star} / b^{\star}\right)^{2}$ & Chroma & hue \\
\hline Junho & $67,0 \pm 2,8$ & $37,1 \pm 0,4$ & $20,3 \pm 0,4$ & $16,1 \pm 0,7$ & $1,3 \pm 0,1$ & $1,6 \pm 0,2$ & $25,9 \pm 0,5$ & $1,0 \pm 0,1$ \\
\hline Agosto & $61,0 \pm 2,3$ & $36,9 \pm 0,4$ & $19,8 \pm 0,7$ & $17,3 \pm 0,8$ & $1,1 \pm 0,1$ & $1,3 \pm 0,2$ & $26,3 \pm 0,5$ & $0,8 \pm 0,1$ \\
\hline Outubro & $59,6 \pm 1,1$ & $37,1 \pm 0,5$ & $18,6 \pm 1,0$ & $16,7 \pm 0,8$ & $1,1 \pm 0,1$ & $1,2 \pm 0,0$ & $25,0 \pm 1,1$ & $0,8 \pm 0,1$ \\
\hline Dezembro & $45,7 \pm 1,4$ & $38,0 \pm 0,5$ & $17,4 \pm 0,9$ & $16,3 \pm 0,8$ & $1,1 \pm 0,1$ & $1,1 \pm 0,0$ & $23,8 \pm 0,2$ & $0,7 \pm 0,1$ \\
\hline Janeiro & $93,5 \pm 0,3$ & $39,0 \pm 0,3$ & $20,7 \pm 0,6$ & $17,7 \pm 0,6$ & $1,2 \pm 0,1$ & $1,4 \pm 0,2$ & $27,2 \pm 0,3$ & $0,9 \pm 0,1$ \\
\hline Março & $28,4 \pm 9,5$ & $41,1 \pm 0,4$ & $16,6 \pm 0,7$ & $19,1 \pm 0,7$ & $0,9 \pm 0,1$ & $0,8 \pm 0,0$ & $25,3 \pm 0,5$ & $0,5 \pm 0,1$ \\
\hline
\end{tabular}

aMédia e desvio padrão de duplicatas.

Em estudos com tomate in natura, foi obtida uma alta correlação $(0,92)$ entre os valores de cor razão a/b e conteúdo de licopeno em tomate [34]. Correlações semelhantes foram encontradas para $L^{*}(0,93), a^{*}(0,96), a^{*} / b^{*}(0,96)$ e conteúdo de licopeno (medido por CLAE), utilizando regressão exponencial, em tomate hidropônico [2].

\section{4 - CONCLUSÕES}

No presente estudo, um método simples foi desenvolvido e otimizado através de planejamento experimental fatorial para extração de licopeno a partir de tomate, consistindo de uma etapa prévia para retirada de água com 4 extrações de 30 minutos cada com $30 \mathrm{~mL}$ de etanol, seguido por 4 extrações com acetato de etila de 120 minutos cada e relação massa/volume de 1:0,7. O teor médio de carotenóides (calculado como licopeno) obtido foi de 59,2 $\pm 21,8 \mu \mathrm{g} / \mathrm{g}$ de descarte tomate. Dos parâmetros de cor, o índice de saturação vermelho ( $\mathrm{a}^{*}$ ) foi o que mostrou melhor correlação com a concentração de licopeno.

Utilizando etapa de baixo custo, foi obtido padrão de licopeno com pureza de $98 \%$ através de cristalização e os cristais formados apresentaram formas irregulares alongadas e prismáticas. A análise por CLAE mostrou a presença de traços dos isômeros 5-cis-, 5-cis, 5'cis- e 13-cis-licopeno no cristal.

\section{5 - REFERÊNCIAS BIBLIOGRÁFICAS}

[1] ASSOCIATION OF OFFICIAL ANALYTICAL CHEMISTS (AOAC). Official methods of analysis. $15 \mathrm{ed}$. Whashington, 1990. p 915-988.

[2] ARIAS, R.; LEE, T. C.; LOGENDRA, L.; JANES, H. Correlation of lycopene measured by HPLC with the $L^{*}, a^{*}, b^{*}$ color readings of a hydroponic tomato and the relationship or maturity with color and lycopene content. J. Agric. Food Chem., v. 48, n. 5, p. 16971702. 2000.

[3] ASSUnÇÃO, R. B.; MERCADANTE A. Z. Carotenoids and ascorbic acid from cashew apple (Anacardium occidentale L.): variety and geographic effects. Food Chem., v. 81, n. 4, p. 495-502. 2003.

[4] BÖHM, V. Use of column temperature to optimize carotenoid isomer separation by $\mathrm{C}_{30}$ high performance liquid chromatography. J. Sep. Sci., v. 24 , n. 12, p. 955-959. 2001.
[5] BÖHM, V; PUSPITASARI-NIENABER, N. L.; FERRUZZI, M. G.; SCHWARTZ, S. J. Trolox equivalent antioxidant capacity of different geometrical isomers of a-carotene, b-carotene, lycopene, and zeaxanthin. J. Agric. Food Chem., v. 50, n. 1, p. 221-226. 2002.

[6] BRITTON, G.; LIAAEN-JENSEN, S.; PFANDER, H. Carotenoids today and challenges for the future. In.: BRITTON G., LIAAEN-JENSEN S., PFANDER H. (Ed) Carotenoids: Isolation and Analysis. Basel: Birkhäuser, 1995. V. 1A. Cap. 2, p. 13-26.

[7] BRITTON, G. UV/visible Spectroscopy. In.: BRITTON G., LIAAEN-JENSEN S., PFANDER H. (Ed) Carotenoids: Spectroscopy. Basel: Birkhäuser, 1995. V. 1B, Cap. 2, p. 13-62.

[8] CEREZAL, P.; PIÑERA, R. M. Carotenoides en las frutas citricas. Generalidades, obtención a partir de desechos del processamento e aplicaciones. Alimentaria, v. 33, n. 277, p. 19-32. 1996.

[9] DAVIES, B. H. Carotenoids. In: Goodwin T. W. (Ed.) Chemistry and Biochemistry of Plant Pigments. London: Academic Press, 1976. V. 2. Cap. 19. p. 38165.

[10] EMENHISER, C.; SANDER, L. C.; SCHWARTZ, S. J. Capability of a polymeric $\mathrm{C}_{30}$ stationary phase to resolve cis-trans carotenoids isomers in reverse-phase liquid chromatography. J. Chromatogr. A, v. 707, n. 2, p. 205-216. 1995.

[11] FERRUZZI, M. G.; NGUYEN, M. L.; SANDER, L. C.; ROCK, C. L.; SCHWARTZ, S. J. Analysis of lycopene geometrical isomers in biological microsamples by liquid chromatography with coulometric array detection. J. Chromatogr. B, v. 760, n. 2, p. 289-299. 2001.

[12] GANN, P. H.; GIOVANNUCCI, E.; WILLET, W.; SACKS, F. M.; HENNEKENS, C. H.; STAMPFER, M. J. Lower prostate cancer risk in men with elevated plasma lycopene levels: results of a prospective analysis. Cancer Res., v. 59, n. 6, p. 1225-1230. 1999.

[13] GAZIEV, A. I. Method of lycopene preparation. Russ Pat. RU 2172608 C1. 2000.

[14] GIOVAnNUCCI, E.; ASCHERIO, A.; RIMM, E. B.; STAMPFER, M. J.; COLDITZ, G. A.; WILlET, W. C. Intake of carotenoids and retinal in relation to prostate cancer risk. J. Nat1. Cancer Inst., v. 87, n. 23, p. 1767-1776. 1995.

[15] GIOVANNUCCI, E. Tomatoes, tomato-based products, lycopene, and cancer: review of the epidemiologic literature. J. Natl. Cancer Inst., v. 91, n. 4, p. 317 331. 1999.

[16] GÓMEZ-PRIETO, M. S.; CAJA, M. M.; HERRAIZ, M. E.; SANTA-MARIA, G. Supercritical fluid extration of 
all-trans-lycopene from tomato. J. Agric. Food Chem., v. 51, n. 1, p. 3-7. 2003.

[17] HAKALA, S. H.; HEINONEN, I. M. Chromatographic purification of natural lycopene. J. Agric. Food Chem., v. 42, n. 6, p. 1314-1316. 1994.

[18] HEINONEN, M. I.; OLLILAINEN, V.; LINKOLA, E. K.; VARO, P. T.; KOIVISTOINEN, P. E. Carotenoids in Finnish foods: vegetables, fruits, and berries. J. Agric. Food Chem., v. 37, n. 3, p. 655-659. 1989.

[19] HENGARTNER, U.; BERNHARD, K.; MEYER, K.; ENGLERT, G.; GLINZ, E. Synthesis, isolation, and NMR-Spectroscopic characterization of fourteen $(Z)$ isomers of lycopene and of some acetylenic didehydroand tetradehydrolycopenes. Helv. Chim. Acta, v.75, n. 6, p. 1849-1865. 1992.

[20] KRINSKY, N. I. The biological properties of carotenoids. Pure Appl. Chem., v. 66, n. 5, p. 1003 -1010. 1994.

[21] LEE, M.T.; CHEN, B.H. Separation of lycopene and its cis isomers by liquid chromatography. Chromatographia, v. 54, n. 9/10, p. 613-617. 2001.

[22] MERCADANTE, A. Z.; RODRIGUEZ-AMAYA, D. B. Carotenoid composition of a leafy vegetable in relation to some agricultural variables. J. Agric. Food Chem., v. 39, n. 6, p. 1094-1097. 1991.

[23] MERCAdAnTe, A. Z.; STECK. A.; PFANDER, H. Carotenoids from guava (Psidium guajava L.): isolation and structure elucidation. J. Agric. Food Chem., v. 47, n. 1, p. 145-151. 1999.

[24] MilleR, E. C.; HADLEY, C. W.; SCHWARTZ, S. J.; ERDMAN, J. W.; BOILEAU, T. W. M.; CLINTON, S. K. Lycopene, tomato products, and prostate cancer prevention. Have we established causality? Pure Appl. Chem., v. 74, n. 8, p. 1435 -1441. 2002.

[25] RE, R.; FRASER, P. D.; LONG, M.; BRAMLEY, P. M.; RICE-EVANS, C. Isomarization of lycopene in the gastric milieu. Biochem. Biophys. Res. Commun., v. 281, n. 2, p. 576-581. 2001.

[26] SANTAMARIA, R. I.; REYES-DUARTE, M. D.; BÁRZANA, E.; FERNANDO, D.; GAMA, F. M.; MOTA, M.; LÓPEZMUNGUÍA, A. Selective enzyme-mediated extraction of capsaicinoids and carotenoids from chili guajillo puya (Capsicum annum L.) using ethanol as solvent. J. Agric. Food Chem., v. 48, n. 7, p. 3063-3067. 2000.

[27] SCHIERLE, J.; BRETZEL, W.; BUHLER, I.; FACCIN, N.; HESS, H.; STEINER, K.; SCHUEP, W. Content and isomeric ratio of lycopene in food and human blood plasma. Food Chem., v. 59, n. 3, p. 459-465. 1997.

[28] SHARMA, S. K.; MAGUER, M. L. Kinetics of lycopene degradation in tomato pulp solids under different processing and storage conditions. Food Res. Int., v. 29, n. 3/4, p. 309-315. 1996.

[29] SHI, J.; MAGUER, M. L.; KAKUDA, Y.; LIPTAY, A.; NIEKAMP, F. Lycopene degradation and isomerization in tomato dehydration. Food Res. Int., v. 32, n. 1, p. 15-21. 1999.

[30] SHI, J.; MAGUER, M. L. Lycopene in tomatoes: chemical and physical properties affected by food processing. Crit. Rev. Food Sci. Nutr., v. 40, n. 1, p. 1-42. 2000.

[31] Statsoft Inc. STATISTICA for Windows [Computer program manual]. Statsoft, Inc.: Tulsa, USA. 1995.

[32] TAVARES, C. A.; RODRIGUEZ-AMAYA, D. B. Carotenoid composition of Brazilian tomatoes and tomato products. Lebensm-Wiss u-Technol., v. 27, n. 3, p. 219-224. 1994.

[33] VAN DEN BERG, H.; FAULKS, R.; GRANADO, H. F.; HIRSCHBERG, J.; OLMEDILLA, B.; SANDMANN, G.; SOUTHON, S.; STAHL, W. The potential for the improvement of carotenoid levels in foods and the likely systemic effects. J. Sci. Food Agric., v. 80, n.7, p. 880-912. 2000.

[34] YANG, C. C.; BRENNAN, P.; CHINNAN, M. S.; SHEWFELT, R. L. Characterization of tomato ripening process as influenced by individual seal-packaging and temperature. J. Food Quality, v.10, n. 1, p. 21-23. 1987.

\section{6 - AGRADECIMENTOS}

Os autores agradecem à FAPESP pela bolsa da primeira autora e auxílio financeiro, e à Roche Vitamins (Basel, Suiça) pela doação dos padrões. 